\title{
Coupled-wave formalism for optical waveguiding by transverse Bragg reflection
}

\author{
Amnon Yariv \\ Department of Applied Physics, California Institute of Technology, Pasadena, California 91125
}

Received January 30, 2002

\begin{abstract}
A formalism is presented for optical waveguiding by means of Bragg reflection. The theory provides analytic expressions for field modal profiles, dispersion, and attenuation. Waveguiding in line defects of photonic bandgap crystals, a special case of the theory, is used as an example. Quantized allowed widths of the guiding channel are predicted. (C) 2002 Optical Society of America
\end{abstract}

OCIS codes: $230.7370,230.1480,130.2790$.

Optical waveguiding based on one-dimensional Bragg reflection rather than total internal reflection has been proposed by Yeh and Yariv ${ }^{1}$ and demonstrated by Cho et al. ${ }^{2} \quad$ Fibers based on transverse circular Bragg confinement were also considered. ${ }^{3}$ More-recent activity in the field of photonic crystals has included considerations and demonstrations of guiding in line defects of such structures. ${ }^{4,5}$ The work to date in the photonic crystal field has relied mostly on numerical simulations. ${ }^{6}$

In this Letter a coupled-mode analysis of optical waveguiding by transverse Bragg reflection is provided that includes as a special case line defects in twodimensional photonic crystals. The model treated here is illustrated in Fig. 1. A guiding channel (GC) with an index $n_{0}$ is bounded on either side by an upper and a lower periodic medium. The upper and lower two-dimensional periodic media (2DPM) bounding the GC can, in general, be different from each other and might possess different symmetries and different unit cell dimensions. Their indices of refraction are represented by $n_{u}(\mathbf{r})$ and $n_{l}(\mathbf{r})$, respectively. Consider the case in which the refractive-index modulation is small compared with the average index so that the field satisfies the scalar Helmholtz equation ${ }^{7}$

$$
\nabla^{2} \mathbf{E}+\omega^{2} \mu \epsilon_{0} n^{2}(\mathbf{r}) \mathbf{E}=0 .
$$

We are looking for the existence of modes confined in the $x$ direction that propagate along the $z$ axis of the GC. Assuming that $\partial / \partial y=0$, such modes should possess the form $f(x) \exp (-i \beta z)$. Wave equation (1) in the GC is satisfied by either harmonic or plane-wave solutions. We take it to be the form of two intersecting plane waves propagating at an equal angle to the $z$ axis:

$$
\begin{aligned}
E_{|x| \leq W / 2}= & E_{i} \exp \left[-i\left(k_{\perp} x+\beta z\right)\right] \\
& +E_{r} \exp \left[-i\left(-k_{\perp} x+\beta z\right)\right],
\end{aligned}
$$

where $E$ represents a principal field component, say, $E_{y} . \quad E_{i}$ and $E_{r}$ are constants. For a self-consistent solution, the field amplitudes $E_{i}$ and $E_{r}$ are not independent, since they are reflected into each other at the upper and lower interfaces. To investigate the conditions for such mutual reflection we need to solve wave equation (1) in the periodic media. We take advantage of the two-dimensional periodicity of the index of refraction to write it as

0146-9592/02/110936-03\$15.00/0

$$
\begin{gathered}
n^{2}(x, z)=\sum_{l, m}\left(n^{2}\right)_{l, m} \exp \left(i \mathbf{K}_{l, m} \cdot \mathbf{r}\right) \\
\mathbf{K}_{l, m}=l \frac{2 \pi}{b} \hat{x}+m \frac{2 \pi}{a} \hat{z} \\
\left(n^{2}\right)_{l, m}=\frac{1}{a b} \int_{-b / 2}^{b / 2} \int_{-a / 2}^{a / 2} n^{2}(x, z) \exp \left(-i \mathbf{K}_{l, m} \cdot \mathbf{r}\right) \mathrm{d} x \mathrm{~d} z
\end{gathered}
$$

where $a$ and $b$ are the dimensions of the primitive unit cell in the $z$ and $x$ directions, respectively. Consistent with the assumption of small index modulation, we take the propagation field vectors in the 2DPM to be the same as in the GC:

$$
\mathbf{k}_{i} \equiv k_{\perp} \hat{x}+\beta \hat{z}, \quad \mathbf{k}_{r}=-k_{\perp} \hat{x}+\beta \hat{z}
$$

and express the field as

$$
E(x, z)=E_{i}(x) \exp \left(-i \mathbf{k}_{i} \cdot \mathbf{r}\right)+E_{r}(x) \exp \left(-i \mathbf{k}_{r} \cdot \mathbf{r}\right) .
$$

Using $\beta^{2}+{k_{\perp}}^{2}=\omega^{2} \mu \epsilon_{0}\left(n^{2}\right)_{0,0}$ and the slowly varying amplitude assumption, $\partial^{2} / \partial x^{2} \ll k_{\perp} \partial / \partial x$, leads to

$$
\begin{aligned}
-2 i k_{\perp} \frac{\partial E_{i}}{\partial x} & \exp \left(-i \mathbf{k}_{i} \cdot \mathbf{r}\right)+2 i k_{\perp} \frac{\partial E_{r}}{\partial x} \exp \left(-i \mathbf{k}_{r} \cdot \mathbf{r}\right) \\
= & -\omega^{2} \mu \epsilon_{0} \sum_{l, m \neq 0}\left(n^{2}\right)_{l, m} \exp \left(i \mathbf{K}_{l, m} \cdot \mathbf{r}\right) \\
& \times\left[E_{i} \exp \left(-i \mathbf{k}_{i} \cdot \mathbf{r}\right)+E_{r} \exp \left(-i \mathbf{k}_{r} \cdot \mathbf{r}\right)\right] .
\end{aligned}
$$

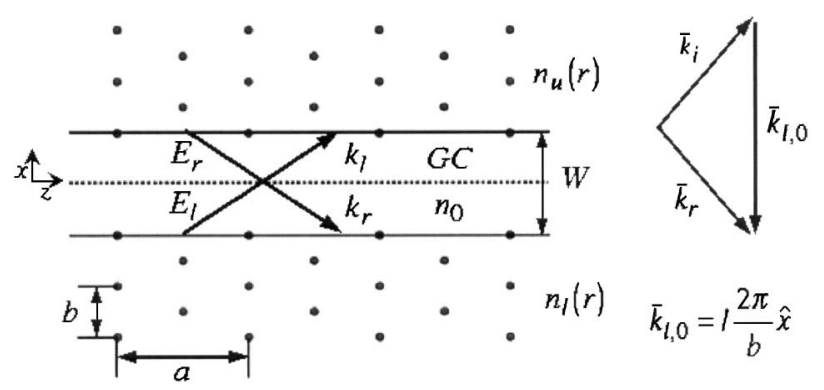

Fig. 1. Triangular array of holes bounding a clear GC as the model used in the present analysis. 
Using the familiar reasoning of the coupled-wave formalism, ${ }^{7}$ we can by proper choice of the reciprocal space vectors $\mathbf{K}_{l, m}$ ensure that each of the two terms on the left-hand side of Eq. (8) is driven by a single term, on the right-hand side, a term with the same exponent. This happens when

$$
\mathbf{k}_{r}-\mathbf{k}_{i}= \pm \mathbf{K}_{l, m}, \quad \mathbf{k}_{i}=\left(k_{\perp}, \beta\right), \quad \mathbf{k}_{r}=\left(-k_{\perp}, \beta\right)
$$

for some pair $l, m$. The crystal momentum conservation condition (9) is shown in the triangle diagram of Fig. 1. Since $\mathbf{k}_{r}$ and $\mathbf{k}_{i}$ have the same $z$ component $(\beta)$, their difference is parallel to $\hat{x}$. Using Eq. (4) restricts the set $\mathbf{K}_{l, m}$ to $\mathbf{K}_{l, 0}$. In this case Eq. (8) reduce to the coupled set

$$
\begin{gathered}
\frac{\partial E_{i}}{\partial x}=\kappa_{l, 0} E_{r}, \quad \frac{\partial E_{r}}{\partial x}=\kappa_{l, 0}^{*} E_{i}, \\
\beta^{2}=\left[(\omega / c)^{2}\left(n^{2}\right)_{0,0}-\left(l \frac{\pi}{b}\right)^{2}\right]^{1 / 2}, \quad l=1,2, \ldots
\end{gathered}
$$

In Eq. (11) we use the momentum condition (see the triangle vector diagram of Fig. 1) to write $k_{\perp}=l \pi / b$. The two-dimensional propagation problem in the 2DPM is thus reduced to two coupled-wave equations (10) involving a single coordinate $x$ and a dispersion relation (11). Equations (10) are identical to those describing a one-dimensional grating. We can thus write their solution directly as ${ }^{6}$

$$
\begin{aligned}
r_{\text {upper }} & =\left.\frac{E_{r}(x)}{E_{i}(x)}\right|_{x=+W / 2} \\
& =-\left(\frac{\kappa_{l, 0}^{*}}{\mid \kappa_{l, 0}}\right)_{\text {upper }} \tanh \left(\left|\kappa_{l, 0}\right|_{\text {upper }} L\right), \\
r_{\text {lower }} & =\left.\frac{E_{i}(x)}{E_{r}(x)}\right|_{x=-W / 2} \\
& =-\left(\frac{\kappa_{l, 0}^{*}}{\mid \kappa_{l, 0}}\right)_{\text {lower }} \tanh \left(\left|\kappa_{l, 0}\right|_{\text {lower }} L\right),
\end{aligned}
$$

where $L$ is the thickness of the 2DPM in the $x$ direction. In Eqs. (12) we allowed for cases in which the upper and lower 2DPM are not identical.

The 2DPM can be fabricated by a variety of techniques, such as ion mixing, indiffusion and outdiffusion, and, in the case of layered semiconductor structures, interface corrugation and regrowth. The coupling coefficients, $\kappa_{l, 0}$, depend on only the Fourier coefficients $\left(n^{2}\right)_{l, 0}$ and not on the specific method employed in the two-dimensional patterning. In the following discussion the example of hole arrays is used.

To complete the solution we use the reflectances $r_{\text {upper }}$ and $r_{\text {lower }}$, Eqs. (12), as boundary conditions on field (2) of the guiding channel at $x=W / 2$ and $x=-W / 2$. This requires that we consider some specific example. We choose the case of a triangular lattice of holes as shown in Fig. 1. We take our holes to be small compared with $a$ or $b$ so that, from Eq. (5),

$$
\begin{aligned}
\left(n^{2}\right)_{l, 0}= & \frac{1}{a b} \int_{-b / 2}^{b / 2} \int_{-a / 2}^{a / 2}-\left(n_{0}^{2}-1\right) A_{\text {hole }}[\delta(x) \delta(z) \\
& \left.+\delta\left(x-\frac{b}{2}\right) \delta\left(z-\frac{a}{2}\right)\right] \exp \left(-i l \frac{2 \pi}{b} x\right) \mathrm{d} x \mathrm{~d} z \\
= & -\frac{\left(n_{0}^{2}-1\right) A_{\text {hole }}}{a b}[1+\exp (-i l \pi)]
\end{aligned}
$$

where $A_{\text {hole }}$ is the hole area. It follows that

$$
\left(n^{2}\right)_{1,0}=0, \quad\left(n^{2}\right)_{2,0}=\frac{-2\left(n_{0}^{2}-1 A_{\text {hole }}\right.}{a b},
$$

so the first nonvanishing reflection is due to $l=2$. Since $\left(n^{2}\right)_{2,0}$ is independent of the $z$ coordinate of the central hole, it follows that the triangular lattice with a lattice constant $b$ of Fig. 1 is equivalent, for our purposes, to a rectangular lattice of row spacing $b / 2$. From Eqs. (11) and (14) it follows that

$$
\kappa_{2,0}=i \frac{2 \pi\left(n_{0}^{2}-1\right) A_{\text {hole }}}{a \lambda_{0}^{2}},
$$

which when used in Eqs. (12) gives

$$
r=i \tanh \left(\left|\kappa_{2,0}\right| L\right) \text {. }
$$

To complete our solution we require that the incident and the reflected fields at the upper $(x=W / 2)$ and lower $(x=-W / 2)$ interfaces of the GC satisfy reflectance conditions (12). From Eq. (2) it follows that

$$
\frac{E_{r} \exp \left(i k_{\perp} W / 2\right)}{E_{i} \exp \left(-i k_{\perp} W / 2\right)}=r, \quad \frac{E_{i} \exp \left(i k_{\perp} W / 2\right)}{E_{r} \exp \left(-i k_{\perp} W / 2\right)}=r,
$$

so that

$$
r^{2} \exp \left(-i 2 k_{\perp} W\right)=1 .
$$

We assume, for the moment, that $|\kappa| L \gg 1$, so that $|r| \approx 1$, and concentrate on the phase condition part of Eqs. (17):

$$
2 k_{\perp} W-2 \theta_{r}=s 2 \pi, \quad s=1,2, \ldots, \quad r \equiv|r| \exp \left(i \theta_{r}\right) .
$$

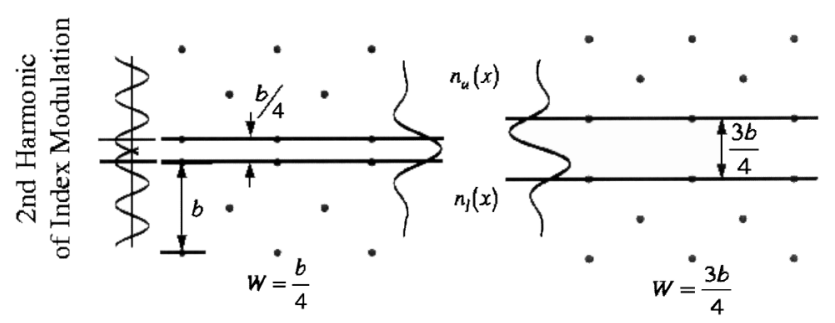

Fig. 2. Transverse modal field distributions for the lowest-order low-loss modes of the structure of Fig. 1 . Note that these are two physically distinct waveguides with different GC widths. 
The second of Eqs. (17) has the exact same form as that of a laser oscillation condition for a wave bouncing vertically in the GC between the 2DPM. ${ }^{7}$ This equation states that a wave returns to the starting plane after a round trip with the same amplitude and the same phase modulo $2 \pi$.

If in the first of Eqs. (18) we use $k_{\perp}=2 \pi / b$ (for the $l=2$ case) as well as $\theta_{r}=\pi / 2$, we obtain

$$
W=\frac{b}{4}, \frac{3 b}{4}, \frac{5 b}{4} \cdots
$$

for the distances between the two rows of holes bordering the GC. Unlike for ordinary dielectric waveguides the width $W$ is quantized and is determined by $b$, diffraction order $l$, and reflectance phase $\theta_{r}$. According to Eq. (19) the usual practice of leaving out a row of holes or an integral number of rows does not make for a low-loss waveguide. If the values of $W$ deviate from Eq. (19), the resulting off-Bragg operation lowers $|r|$, thus increasing the modal loss. The spacing $b / 4$ of the lowest-order mode is formally equivalent to the $\lambda / 4$ phase slip of distributed feedback lasers, except that here it involves the transverse dimension $(x)$ rather than the propagation direction $(z)$. We use Eq. (19) in Eq. (2) and the fact that in the limit $|\kappa| L \gg 1$ the field in the 2DPM evanesces as ${ }^{8}$ $\exp (-|\kappa| x)$ to write for case I

$$
\begin{gathered}
W=\frac{b}{2}, \\
E(x, z)=\exp (-i \beta z) \\
\times \begin{cases}E_{0} \cos \left(\frac{2 \pi}{b} x\right) & |x| \leq \frac{b}{8} \\
E_{0} \cos \left(\frac{2 \pi}{b} x\right) \exp \left\{-\left[\left|\kappa_{2,0}\right|\left(x-\frac{b}{8}\right)\right]\right\} & x \geq \frac{b}{8} \\
E_{0} \cos \left(\frac{2 \pi}{b} x\right) \exp \left\{\left[\left|\kappa_{2,0}\right|\left(x+\frac{b}{8}\right)\right]\right\} & x \leq-\frac{b}{8}\end{cases}
\end{gathered}
$$

and for case II

$$
\begin{gathered}
W=\frac{3 b}{4}, \\
E(x, z)=\exp (-i \beta z) \\
\times \begin{cases}E_{0} \sin \left(\frac{2 \pi}{b} x\right) & |x| \leq \frac{3 b}{8} \\
E_{0} \sin \left(\frac{2 \pi}{b} x\right) \exp \left\{-\left[\left|\kappa_{2,0}\right|\left(x-\frac{3 b}{8}\right)\right]\right\} & |x| \geq \frac{3 b}{8} \\
E_{0} \sin \left(\frac{2 \pi}{b} x\right) \exp \left\{\left[\left|\kappa_{2,0}\right|\left(x+\frac{3 b}{8}\right)\right]\right\} & x \leq-\frac{3 b}{8}\end{cases}
\end{gathered}
$$

Solutions (21) possess the Floquet-Bloch form ${ }^{9}$ inside the 2DPM. They are demonstrated in Fig. 2. Natomi et al..$^{10}$ investigated numerically and experimentally transmission in a triangular (hexagonal) lattice. Transmission was observed at GC widths by use of this notation, in the range $W=0.7 b$ to $W=0.8 b$, which brackets our theoretical value of $0.75 b$ of the odd solution ( $W=3 b / 4)$ equations (21). Natomi et $a l .{ }^{10}$ did not report on the symmetry of the mode and did not explore the case of a $W=b / 4$ waveguide, in which the even mode, Eqs. (20), should exist.

In the limit of small index modulation, where the present formalism applies, we can consider two or more periodic structures to be able to share the same space with minimal interference. This can be used to phase match nonlinear interactions.

The exponential propagation loss constant $\alpha$ of the transverse Bragg waveguide modes is obtained as the product of the number of ray bounces per unit length multiplied by the reflection loss per bounce. ${ }^{8}$ For large reflectivity, $\kappa L \gg 1$, we obtain from Eq. (16) an intensity loss constant

$$
\alpha=\frac{16 \pi \exp (-2 \kappa L)}{\beta b^{2}}
$$

for a waveguide GC with a width $W=b / 4$.

In summary, a coupled-wave approach has been applied to obtain closed-form expressions for the properties of traveling-wave modes that are confined transversely by Bragg reflection.

The author is thankful for support of this work by the U.S. Office of Naval Research (Y. S. Park), the Defense Advanced Research Projects Agency (D. Honey and R. Athale), and the U.S. Air Force Office of Scientific Research (H. Scholossberg) and for useful input from his students, J. Choi, S. Mookherjea, and G. T. Paloczi, and critical comments by Bruno Crosignani.

\section{References}

1. P. Yeh and A. Yariv, Opt. Commun. 19, 427 (1976).

2. A. Y. Cho, A. Yariv, and P. Yeh, Appl. Phys. Lett. 30, 471 (1977).

3. P. Yeh, A. Yariv, and E. Marom, J. Opt. Soc. Am 68, 1196 (1978).

4. S. Y. Lin, E. Chow, S. G. Johnson, and J. D. Joanopoulos, Opt. Lett. 25, 1297 (2000).

5. M. Loncar, D. Dedeljkovic, T. Doll, A. Scherer, J. Vuckovic, and T. Pearsall, Appl. Phys. Lett. 25, 1937 (2000).

6. K. Sakoda, Phys. Rev. B 51, 4672 (1995).

7. See, for example, A. Yariv, Optical Electronics in Modern Communication, 5th ed. (Oxford U. Press, Oxford, 1997).

8. A. Yariv and P. Yeh, Optical Waves in Crystals (Wiley, New York, 1984), p. 478.

9. See, for example, N. Ashcroft and D. Mermin, Solid State Physics (Holt, Rinehart \& Winston, New York, 1976), p. 133.

10. M. Notomi, A. Shinya, K. Yamada, J. Takahashi, C. Takahashi, and I. Yokohama, Electron. Lett. 37, 293 (2001). 